\title{
Ocular larva migrans: a case report
}

\author{
ROBERT W LYNESS,' OLIVIA E EARLEY,' WILLIAM C LOGAN,?, \\ AND DESMOND B ARCHER'
}

From the Departments of 'Pathology and 'Ophthalmology, the Queen's University of Belfast, Royal Victoria Hospital, Belfast BT12 6BA

SUMmaRy A case of intraocular toxocara larva migrans occurring in a 12-year-old boy is presented. Motility is inferred from the nature of multiple intraocular lesions observed. Possible mechanisms preventing a conclusive immunological response to the organism are discussed.

Ocular toxocariasis continues to present the ophthalmologist with a problem of diagnosis despite improved investigative tools. Several clinical patterns of presentation are recognised.' One of these, the motile chorioretinal nematode, has been described following ophthalmoscopic visualisation, ${ }^{23}$ and inferred from the discovery of a subretinal tube on histological examination of a presumed case of ocular toxocariasis. ${ }^{+}$We present a case of ocular toxocariasis in a 12-year-old boy which purports to demonstrate the intraocular motility of the toxocara larva and the nature of the various lesions caused by its passage. We relate the difficulty in achieving serological confirmation of ocular toxocariasis to peculiarities in the intraocular host-parasite relationship.

\section{Case report}

A 12-year-old boy first attended the ophthalmic outpatient clinic of the Royal Victoria Hospital, Belfast, on 26 March 1985. He complained of a disturbance of vision in his left eye, present for approximately six weeks.

Three weeks after the onset of symptoms the patient found he was unable to see out of the left eye, and since then there had been no recovery of vision. The affected eye was comfortable. There was no history of injury, ocular inflammation, or discharge. The patient was in good health otherwise, though he suffered from intermittent attacks of asthma which were treated with a salbutamol inhaler. The asthmatic attacks were precipitated occasionally by contact with a pet rabbit. There was no obvious contact with dogs, cats, or farm animals. At the age of 6 years the patient was admitted to hospital suffering from Correspondence to Dr R W Lyness. febrile convulsions. No specific diagnosis was made and convulsions have not recurred. There was no family history of any ocular or relevant systemic disease.

\section{EXAMINATION}

At the first examination, on 26 March 1985, the visual acuity of the unaided right eye was recorded as $6 / 4$ and that of the left eye as accurate projection of light. Examination of the right eye showed it to be structurally sound in all regards. The left eye was injected, and biomicroscopy revealed slight flare and some inflammatory cells in the anterior chamber. Pigment deposits on the anterior lens surface and posterior synechiae were present (Fig. 1). The intraocular pressure was $18 \mathrm{mmHg}$ on the right and $24 \mathrm{mmHg}$ on the left. The left lens was clear, but immediately behind the lens there was a grey-white intraocular membrane or mass associated with an array of dilated vascular channels and pigment deposits. The ocular fundus could not be visualised.

Systemic examination was negative, with no evidence of regional or generalised lymphadenopathy, skin lesions, arthropathy or hepatosplenomegaly.

The right visual field was normal (Goldmann perimetry) but it was not possible to obtain a plot on the left side. Electroretinography showed a normal B wave amplitude on the right side, but recordings on the left side were extinguished, even to a bright flash. Fluorescein angiography (Fig. 2) confirmed the presence of numerous dilated shunt-like blood vessels within the retrolenticular mass. These blood vessels filled rapidly in the early phases of angiography; the major channels remained competent, while the smaller vessels leaked dye, causing the retrolenticular mass to become hyperfluorescent by the later angiographic frames. Some of the large 


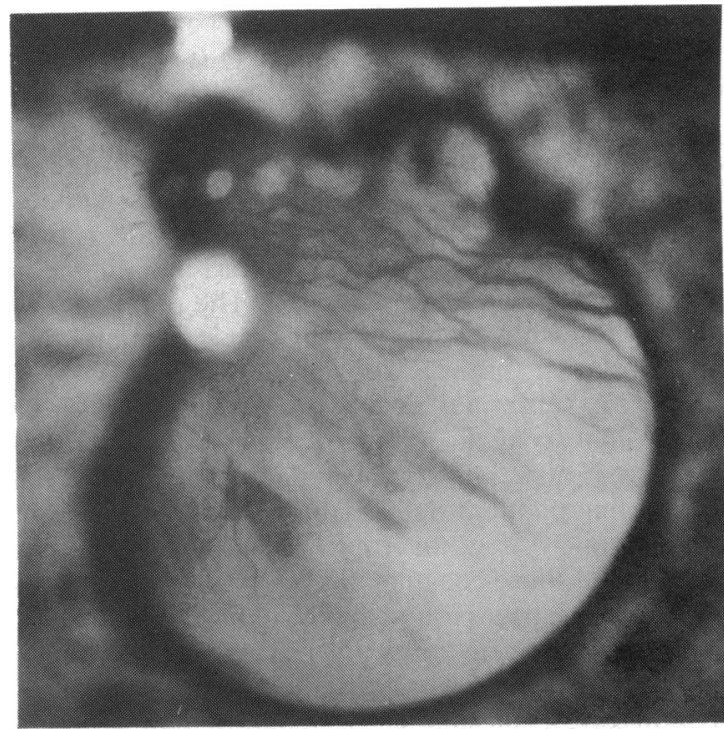

Fig. 1 Biomicroscopic view of iris showing posterior synechiae and the vascular cyclitic membrane.

vessels remained non-perfused throughout the angiographic series, which was consistent with either thrombosis or the 'steal phenomenon' seen in tumour circulations. The late phase angiograms showed leakage of dye at the superior iris, but there was no evidence of rubeosis iridis.

The white blood cell count was $6.5 \times 10^{4} / 1$ and the eosinophil polymorphonuclear count was $0 \cdot 26 \times 10^{\%} / 1$ or $4 \%$ of the differential white cell count. The erythrocyte sedimentation rate (ESR) was $33 \mathrm{~mm}$ in the first hour. The blood alkaline phosphatase at 182 $\mathrm{ml} / \mathrm{l}$; (considered raised in an adult but within normal range for a child of 12 years of age) was the only biochemical finding. The Rose-Waaler test and serological tests for $\mathrm{Rh}$ factor and rheumatoid arthritis factor (latex) were negative. Serological tests for toxoplasmosis were negative. The toxocara antibody enzyme linked immunosorbent assay (ELISA) test was weakly positive $(0.63$ units: normal values $0-0 \cdot 50$ ). Chromosome analysis showed no abnormalities.

$X$-ray examination of the chest showed nothing unusual except for an abnormality of the pulmonary conus, which was not considered significant. $X$-rays of the left orbit were normal, and there was no evidence of intraocular calcification. Ultrasound examination identified a retrolenticular mass consisting of a dense posterior cyclitic membrane and a funnel shaped retinal detachment. CT scanning showed a distinct abnormality of the left eye, the globe appearing to be of increased attenuation, with an area of further increased attenuation noted

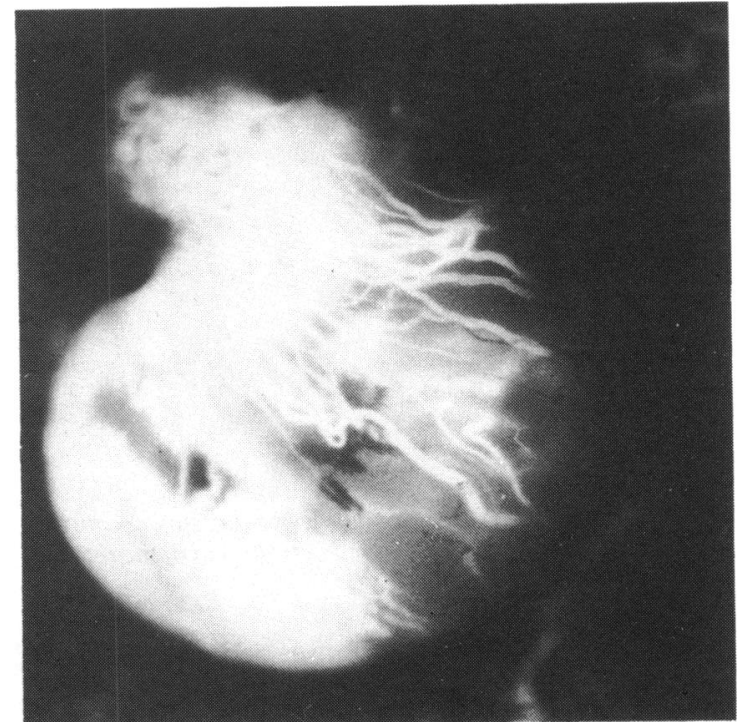

Fig. 2 Late phase fluorescein angiogram showing large vascular channels within retrolental membrane.

posterolaterally. The appearance of the CT scan suggested to the radiologist 'a malignant tumour within the globe which may have arisen from the retina'.

\section{MANAGEMENT}

The patient was followed up for four weeks, during which time the visual acuity diminished to no perception of light in the left eye and the intraocular pressure fell to $4 \mathrm{mmHg}$. In view of the loss of visual function, impending ocular hypotony, and the suggestion of the presence of an intraocular tumour on CT scanning, enucleation of the left eye was carried out on 30 April 1985.

\section{PATHOLOGICAL FINDINGS}

The globe was fixed in formalin prior to processing and embedding in wax. Macroscopically the left eye measured $23 \times 23 \times 22 \mathrm{~mm}$ and showed no gross external abnormality. The cut surface showed an apparently normal anterior chamber. A cyclitic membrane posterior to a normal lens with an adherent funnel shaped detachment of the retina was present.

Histological examination of serial sections showed a normal cornea, filtration angles, iris, and lens. A cyclitic membrane (Fig. 3) extended from one part of the ciliary body, posterior to the lens, to the contralateral aspect of the ciliary body. This membrane consisted of three generations of inflammatory tissue. These showed resolved and resolving inflammation with fibrinous organisation and more recent 


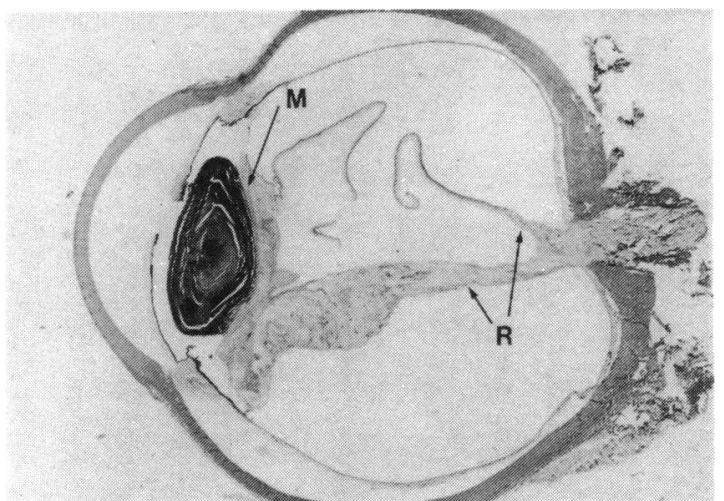

Fig. 3 Histological section of whole eye showing cyclitic membrane $(\mathrm{M})$ and funnel shaped detachment of the retina (R).

inflammation composed of loose, oedematous, serofibrinous material infiltrated mainly by eosinophil polymorphonuclear leucocytes but including some neutrophil polymorphs, lymphocytes, and plasma cells. Large blood vessels were readily apparent. The membrane extended over the inner aspect of the retina producing a tractional retinal detachment.

The retina was well preserved in most areas, which confirmed that the detachment was a recent occurrence. There were two foci (Fig. 4) of eosinophilic necrosis with the features of the Splendore-Hoeppli phenomenon's situated in the outer retina. No organism or remnants of an organism were found in either of these lesions. One lesion contained Charcot-Leyden crystals. Elsewhere in the posterior retina there was perivascular chronic inflammatory cell infiltration.
The choroid contained foci (Fig. 5) of eosinophilic polymorphonuclear inflammation. One of these focal lesions showed a perforated Bruch's membrane partially damming back the eosinophils and necrotic eosinophilic material to cause a dark staining area on the choroidal aspect. Eosinophilic material exuded through Bruch's membrane, detaching the retinal pigment epithelium. No organism or remnants of an organism was found in this lesion. The remainder of the choroid showed low grade chronic inflammation and a relative absence of eosinophils.

In the subretinal space four separate discrete spheroidal coagula (Fig. 6) were found. These consisted of relatively acellular hyaline material containing pigment debris from the retinal pigment epithelium. A few macrophages were seen on the surfaces of these coagula. No organism or remnants of an organism was found in any of these four lesions.

On level 152 of the serial histological sections a granuloma (Figs. 7 and 8) composed mainly of lymphocytes, plasma cells, macrophages, foreign body type giant cells, and a few eosinophils was seen in the subretinal space. This lesion contained a larva of Toxocara canis with characteristic morphological and structural features. ${ }^{6}$

\section{Discussion}

We have demonstrated eight lesions in this eye associated with ocular toxocariasis, raising the question of how many larvae were involved in their production. It is our contention that only one larva rather than up to eight was involved, as only one larva was found after serial sectioning of each of the other seven lesions. There was evidence of sequential
Fig. 4 Histological section of the outer retina showing an area of eosinophilic necrosis $(\mathrm{N})$ with foci of Charcot-Leyden crystals (C-L, arrowed). Also present is a subretinal coagulum (Coag).

$H$ and $E$, polarised light. $\times 52$.

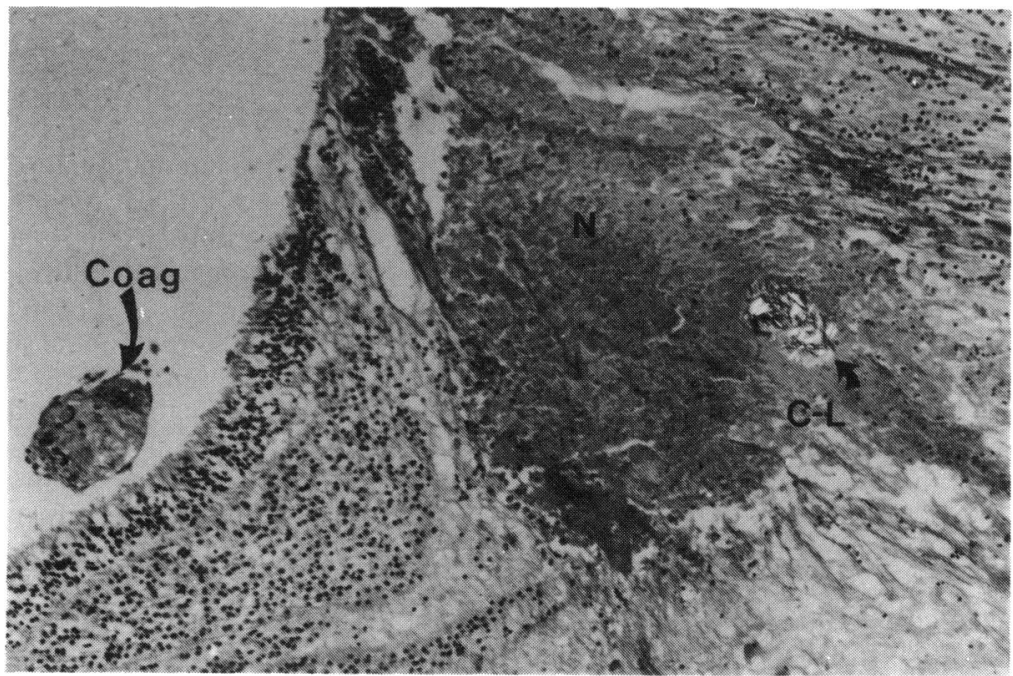




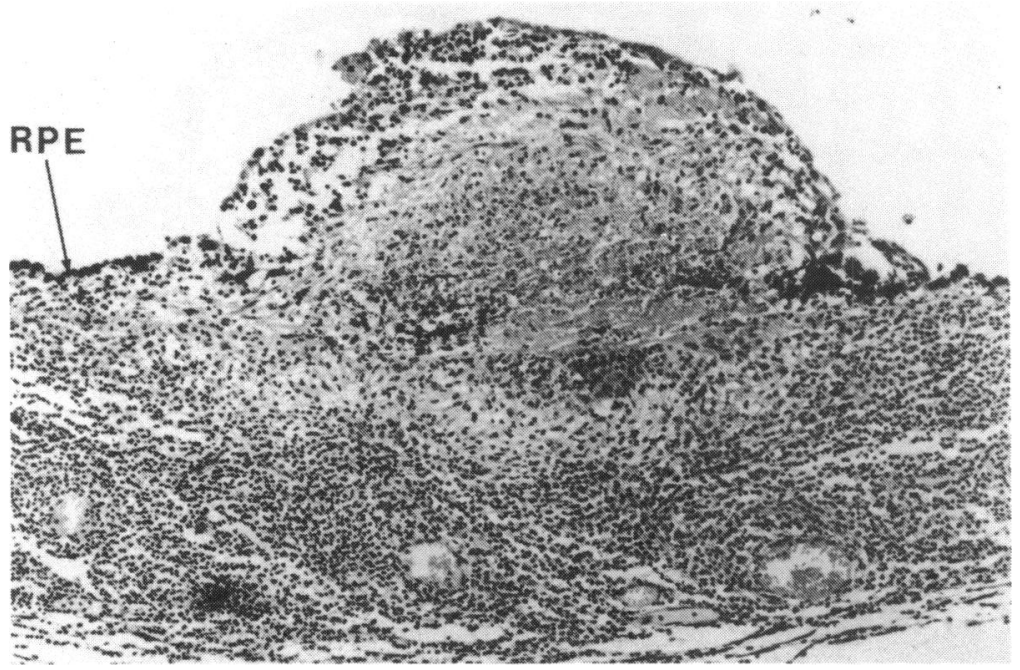

Fig. 5 Histological section of choroid showing an eruption composed mainly of eosinophils and detaching the overlying retinal pigment epithelium (RPE). $H$ and $E . \times 52$.

(rather than synchronous) inflammatory events giving rise to several 'waves' of cyclitic membrane formation and chorioretinal lesions at different stages of development or degeneration. Furthermore, there was no indication of worm infestation of the fellow eye or systemically.

The lesions we have described seem to represent different stages of the immunological reaction by the host to the parasite. The immediate reaction, surrounding the larva, consists mainly of lymphocytes, macrophages, and a few eosinophils. ' In the subjacent choroid, eosinophilic polymorphonuclear leucocytes have accumulated and intensified the focal inflammatory response, eventually erupting through Bruch's membrane to attack the larva within the subretinal space. This choroidal lesion is character- ised by an area of eosinophilic necrosis, surrounded by viable eosinophils, which extrudes through a perforated Bruch's membrane to elevate the overlying retinal pigment epithelium. The choroidal eruption appears to have resolved, giving rise to an expended coagulum as seen in the subretinal space and possibly the Splendore-Hoeppli phenomena.

It is possible to plot the path (Fig. 9) taken by the larva in the vitreous and subretinal space, deducing the chronological order from the type and age of the eight lesions present in this case. The likely sequence of events is that the larva entered the anterior vitreous from a peripheral branch of the retinal artery, initiating the reaction that caused the cyclitic membrane. The topography of the blood vessels identified in the cyclitic membrane suggests that the

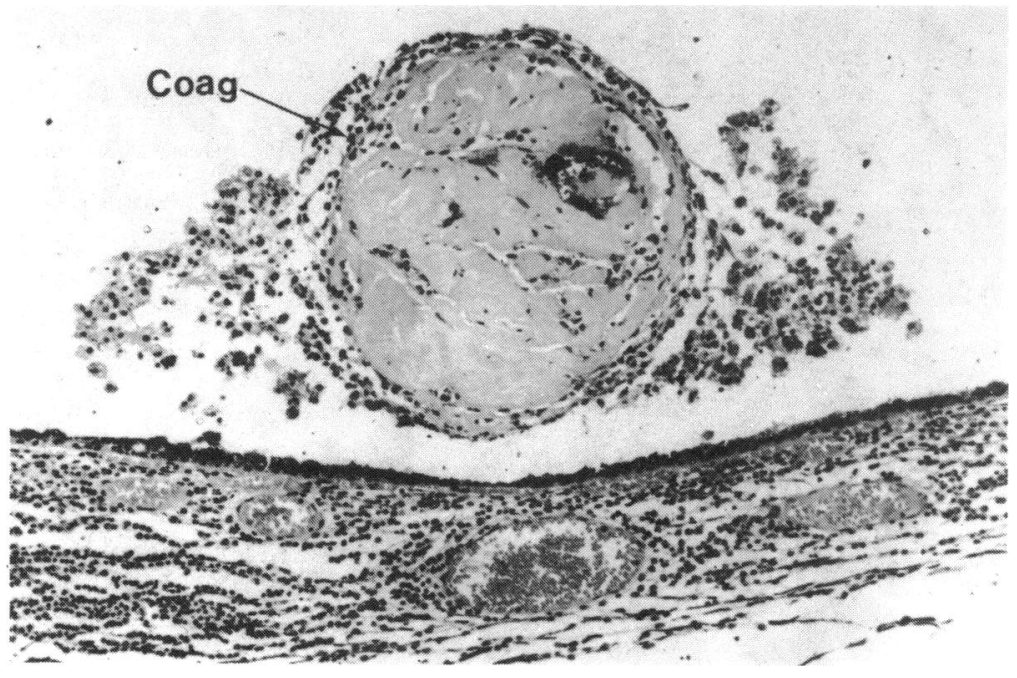

Fig. 6 Histological section of a subretinal coagulum (Coag) surrounded by macrophages and remnants of a choroidal eruption. $H$ and $E . \times 52$. 


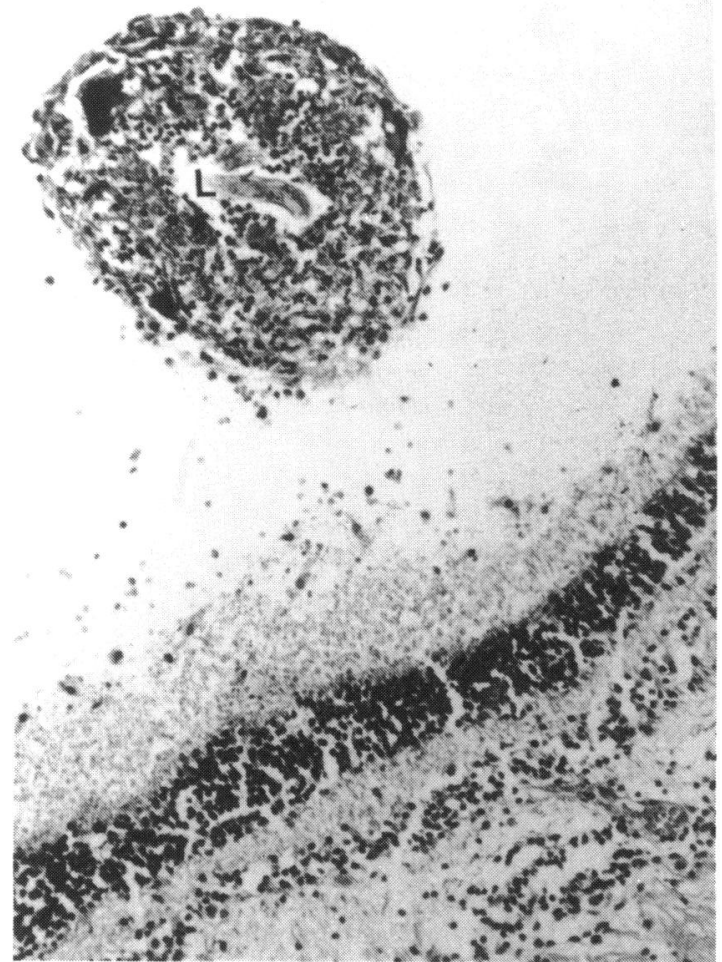

Fig. 7 Histological section of subretinal granuloma (L), which includes a toxocara larva. $H$ and $E . \times 57$.

larva moved from one part of the ciliary body to a contralateral site and then gained access to the subretinal space. Within the subretinal space the larva moved freely. Such motility of the toxocara

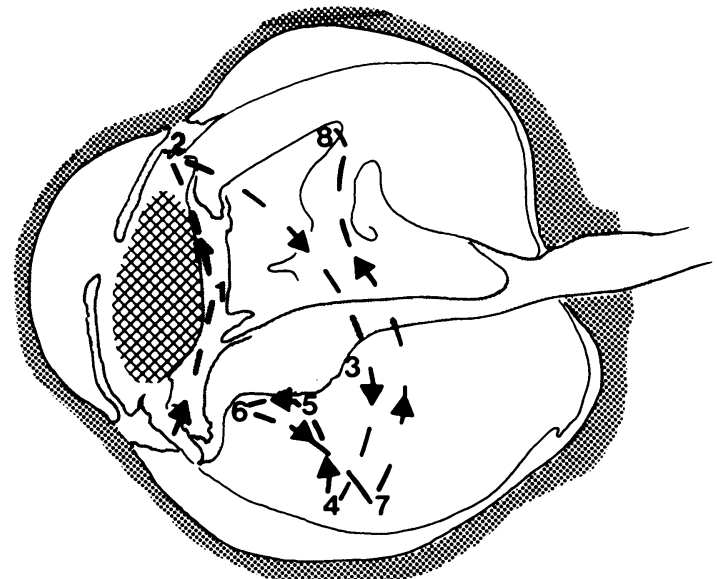

Fig. 9 Drawing of the eye depicting the presumed path of the toxocara larva. (1) Entrance to vitreous from retinal circulation. (2) Site of coagulum at point of entry to subretinal space. $(3,4,5)$ Sites of subretinal coagula. (6) Sites of Splendore-Hoeppliphenomenon. (7) Site of choroidal eruption. (8) Site of subretinal granuloma containing the toxocara larva.

larva and presumed helminths within the eye has been reported in experimental toxocariasis in the eyes of mice ${ }^{x y}$ and primates, ${ }^{10}$ by the presence of a subretinal tube in one case report, ${ }^{4}$ and by clinicians on ophthalmoscopic examination. ${ }^{23}$

This case is an example of the clinical dilemma of differentiating between a malignant intraocular tumour and other benign intraocular lesions despite possession of an array of sophisticated and sensitive
Fig. 8 Histological section of subretinal granuloma showing a toxocara larva (L) and a giant cell (GC), Note the paucity of eosinophils. $H$ and $E . \times 52$

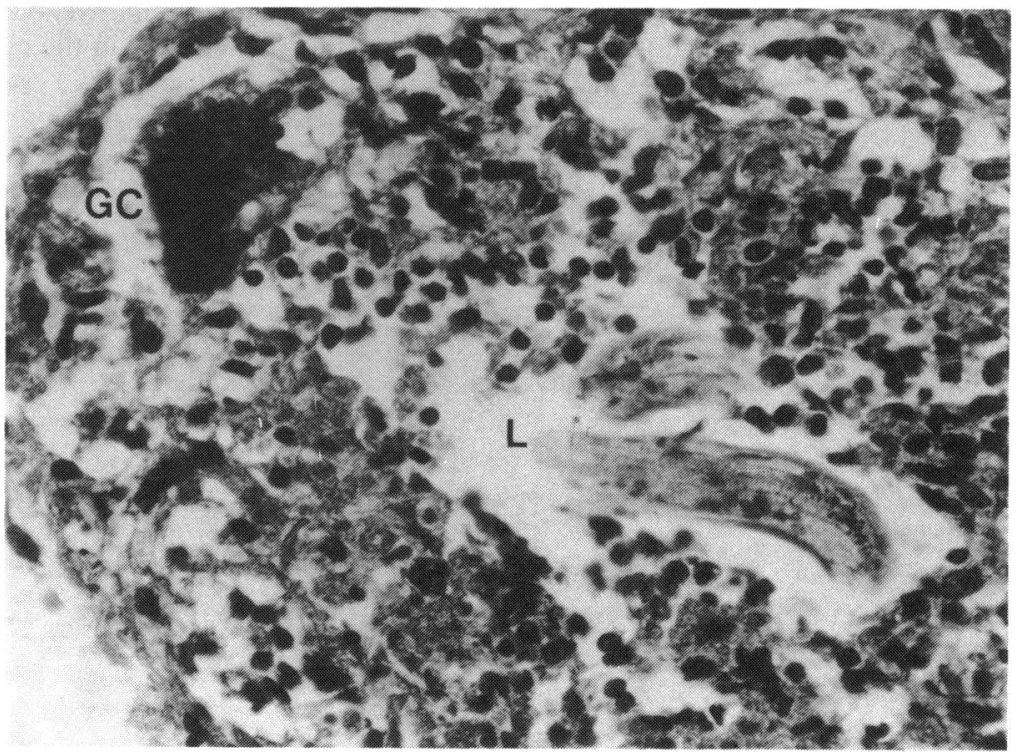


diagnostic tests. Of particular interest is the relatively low eosinophil polymorph count $\left(0 \cdot 26 \times 10^{\prime} / 1\right.$ or $4 \%$ of WBC) in the peripheral blood (normal values in children $\left.0.03-0.7 \times 10^{\prime \prime} / 1\right)^{\prime \prime}$ and the barely positive ELISA titre for toxocara, when compared with the pronounced inflammatory reaction in the eye as seen on histological examination. There is no evidence that this host (in common with the patients in previous reports) ${ }^{12}$ was immunologically compromised or deficient before or after clinical presentation.

Previous reports ${ }^{13}$ support the contention in this case that the worm load in patients with ocular toxocariasis is low. This may result in an appropriately slight immunological response by the host. ${ }^{1+}$ The findings in our case strongly suggest that the larva of toxocara can move away from an inflammatory response to its presence. It is probable that in this process the parasite manipulates its antigenic profile by shedding its 'coat' (glycocalyx) ${ }^{15}$ Thus residual antigenic material continues to stimulate the inflammatory response while the parasite in a new guise moves elsewhere. A change of antigenic profile serves to prevent a concerted antibody response by the host, as each change of surface antigens requires synthesis of a new set of antibodies to combat them. ${ }^{16}$

A further factor in the host-parasite relationship peculiar to the eye may be the immunological surveillance of the subretinal space. The outer retina and the subretinal region are nourished and serviced by the choroidal circulation. It would appear that the initial response via the retinal circulation and the subsequent choroidal reaction may be delayed or curtailed by the inner and outer blood retinal barriers respectively. The evidence from our case suggests that the response to the larva in the subretinal space is a granuloma closely followed by a choroidal eruption consisting predominantly of eosinophils, bursting through Bruch's membrane into the subretinal space in a bid to attack the parasite before it moves on. This obviously works in some instances, as previous case reports have described the remnants of larvae in similar isolated chorioretinal lesions.

This case appears to represent an intermediate stage between a solitary choroidoretinal lesion ${ }^{12}$ and nematode endophthalmitis ${ }^{17}$ in the destruction of an eye by the toxocara larva. The histopathological appearances show that the larva can defend itself by moving within the subretinal space. It is also suggested that the response of the host is attenuated by an inadequate immunological surveillance of the subretinal space with inner and outer blood retinal barriers obstructing a concerted immunological response to the organism. It is perhaps for these reasons and the scant number of parasites that the eosinophil count and ELISA titre are so low despite the presence and activity of the larva. Such a hypothesis suggests that an organism in the eye could easily give rise to a negative or dubious positive result on examination of the peripheral blood. This situation will lead to a continuation of the diagnosis of 'choroidoretinitis' due to presumptive toxocariasis in clinical ophthalmic practice, despite the availability and use of sensitive serological tests.

\section{References}

1 Shiclds JA. Ocular toxocariasis. A review. Surv Ophthalmol 1984; 28: 361-81.

2 Parsons HE. Nematode chorioretinitis: report of a case with photography of a viable worm. Arch Ophthalmol 1952; 47: 799-800.

3 Rubin ML, Kaufman HE, Ticrncy JP. An intrarctinal nematode. Ophthalmology (Rochester) 1968; 72: 855-66.

4 O'Connor PR. Visceral larva migrans of the eyc. Subretinal tube formation. Arch Ophthalmol 1972; 88: 526-9.

5 Garner A, Klintworth GK. Pathology of ocular disease. New York, Basel: Dekker, 1982: 364.

6 Nichols RL. The etiology of visceral larva migrans. J Parasitol 1956; $42: 349$.

7 Kayes SG, Oaks JA. Development of the granulomatous response in murine toxocariasis. Am J Pathol 1978; 93: 277-94.

8 Olson LJ. Ocular toxocariasis in micc: distribution of larvac and lesions. Int J Parasitol 1976; 6: 247-51.

9 Ghafoor SYA, Smith HV, Lec WR, et al. Experimental ocular toxocariasis: a mouse model. Br J Ophthalmol 1984; 68: 89-96.

10 Watzke RC, Oaks JA, Folk JC. Toxocariasis infection of the cyc. Correlation of clinical observations with developing pathology in the primate model. Arch Ophthalmol 1984: 102: 282-91.

11 Orfanakis NG, Ostlund RE, Bishop CR, et al. Normal blood lcukocytc concentration values. Am J Clin Pathol 1970; 53: 647.

12 Ashton N. Toxocara canis and the cyc. Br J Ophthalmol 1960; 44: 129-46.

13 Glickman LT, Schantz PM. Epidemiology and pathogenesis of zoonotic toxocarisis. Epidemiol Rev 1981; 3: 230-50.

14 Wiseman RA. Hepatomegaly and allergic responses in helminthic infections transmitted from animals. Proc $R$ Soc Med 1969; 62: 1046-8.

15 Smith HV, Quinn R, Kusel JR, et al. Effects of temperature and antimetabolites on antibody binding to outer surface of second stage Toxocara canis larvac. Mol Biochem Parasitol 1981; 4: 183-93.

16 Rockcy JH, Donnclly JJ, Stromberg BE. Immunopathology of ascarid infection of the cyc. Arch Ophthalmol 1981; 99: 1831-40.

17 Duguid IM. Chronic endophthalmitis due to toxocara. Br J Ophthalmol 1961; 45: 705-17.

Accepted for publication 14 July 1986. 